\title{
Comparison of Abdominal Muscle Thickness Using Ultrasound Imaging During Bridging Exercises With a Sling and Ball in Healthy Young Adults
}

\author{
Young Moon ${ }^{1,2}, \mathrm{MSc}, \mathrm{PT}$, Jong-duk Choi², PhD, PT \\ ${ }^{1}$ Department of Movement Development, The ERUM Child Development Center, ${ }^{2}$ Department of Physical Therapy, College of Health and \\ Medical Science, Daejeon University, Daejeon, Korea
}

\author{
Article Info \\ Received October 1, 2019 \\ Revised November 2, 2019 \\ Accepted January 7, 2020 \\ Corresponding Author \\ Jong-duk Choi \\ E-mail: choidew@dju.kr \\ https://orcid.org/0000-0002-9663-4790
}

\section{Key Words}

Exercise therapy

Stability

Sling

Ultrasonography
Background: Bridging exercises are used to enhance the functional stability of the lumbopelvic region in clinical settings. Although most of the studies on bridging exercises have compared the complete activation of the trunk muscles, some recent studies have examined the functional stability of the trunk and the lumbopelvic region and assessed the appropriate recruitment of the local and global muscles during different task levels.

Objects: The purpose of this study was to investigate the changes in muscle thickness in the transverse abdominis (TrA), internal oblique (IO), and external oblique (EO) muscles during a common bridging exercise on an unstable surface and to determine whether these changes differ based on the surface used.

Methods: Twenty-five healthy young adults (8 males, 17 females) were recruited. The subjects were randomly assigned to either the exercise progression with a sling bridge group or the ball bridging exercise progression group, each with three stages of increasing difficulty. Each position was measured three times with an ultrasonic diagnostic imaging system, and the mean values were recorded for analysis.

Results: No significant differences were observed between the $\operatorname{Tr} A$, IO, or EO muscle thickness ratios between the sling and ball exercise groups $(p>0.05)$. There were also no significant differences in the EO muscle thickness ratios between the tasks irrespective of whether the sling or ball was used. However, the TrA and 10 thickness ratios in both groups were significantly greater during stages 2 and 3 compared to stage 1 .

Conclusion: The results suggest that the use of slings and balls during bridging exercises is effective in activating the deep abdominal muscles.

\section{INTRODUCTION}

The stability of the lumbopelvic region and neuromuscular control of the trunk not only provide flexibility of body parts but also prevent musculoskeletal injuries [1]. Abnormal postures, inappropriate muscle activation, and abnormal movements of local muscles lead to loss of control of the lumbopelvic region and serious problems in trunk stability $[2,3]$.

The trunk musculature consists of local muscles and global muscles that play important roles in many functional and athletic activities. Larger and superficially located muscles in the abdominal and back region include global muscles, such as the rectus abdominis, the paraspinalis, and the external oblique $[4,5]$. These muscles are mainly used for trunk or hip flexion, extension, and abduction and contribute to overall trunk stability. Local muscles, such as the transverse abdominis, the internal oblique, and the multifidus, are smaller and deeply located in the abdominal and back region. These are directly connected to spinal segments and involved in fine spinal control and the stability of spinal segmental motions $[5,6]$.

Bridging exercises are used to enhance the functional stability of the lumbopelvic region in clinical settings. Bridging exercises can relieve lumbopelvic pain, make the patient feel more comfortable, and retrain the local muscles and the global muscles to ensure that these muscles can be recruited at appropriate rates [7]. Many studies have examined the effects of bridging exercises on lumbar stability. García-Vaquero et al. [8] compared abdominal muscle activation during bridging 
exercises performed with one leg raised with that during traditional bridging exercises, and Bjerkefors et al. [9] compared EMG activation of the back and the abdominal muscles during bridging exercise in a series of postures.

Unstable surfaces such as Swiss balls, air cushions, and sling suspension systems were used to increase muscle activation and coactivation to enhance the stability of the lumbopelvic region in theprogressing stage $[10,11]$. The results showed that the unstable surfaces were more effective in activating trunkstabilizing muscles than the stable surfaces.

Most of the studies of bridging exercises conducted thus far have compared complete activation of the trunk muscles. Few studies have assessed the appropriate recruitment of the local muscles and the global muscles during different levels of difficulties of tasks to examine the functional stability of the trunk and the lumbopelvic region. In addition, the levels of muscle activation on a series of surfaces and the activation rates of the deep muscles and the superficial muscles have not been measured.

Thus, the purpose of the present study was to examine the thicknesses of the transverse abdominal muscle, the internal oblique muscle, and the external oblique muscle when bridging exercises of different degrees of difficulties were performed on unstable surfaces using sling suspension systems and balls.

\section{MATERIALS AND METHODS}

\section{Subjects}

Twenty-five healthy young persons (8 males and 17 females) who understood the content of the study and voluntarily agreed to participate were recruited. The inclusion criteria were as follows: no experience of low back pain during the last six months, no orthopedic surgery, no neurological illness affecting the lower extremities or the lumbopelvic region, and sufficient muscle strength and range of motion to perform the tasks. The mean age of the subjects was $22.28 \pm 2.82$ years, the mean height was $165.92 \pm 8.63 \mathrm{~cm}$, and the mean weight was $58.84 \pm 9.84 \mathrm{~kg}$.

\section{Ultrasound Imaging}

Prior to enrolling subjects, examiners underwent a supervised training program for the ultrasonic diagnostic imaging system by an experienced physical therapist. The center of the transducer was placed on the transverse plane across the abdominal wall on the axillar line in the middle of the area between the no. Twelve costal cartilage on the right side and the iliac crest to obtain clear images of the deep abdominal layer [12]. To standardize the location of the transducer, the space where the transverse abdominal and the thoracolumbar fascia meet each other was shown at the end of the right side of the images [13]. The angle of the transducer was adjusted to optimize the visualization of the images.

In the present study, the ultrasound images were obtained in B (brightness) mode. To control the effects of breathing cycles on the abdominal muscle thicknesses and ensure that the measurement times were identical among the subjects, the subject maintained the position of each exercise for 5 seconds at the last expiration, which was determined by visual observation of the abdominal region. Three repeated ultrasound images were used to determine the abdominal muscle thicknesses [14].

To determine the abdominal muscle thicknesses from the ultrasound images, the distances between the superior and inferior hyperechoic muscle fascias of the transverse abdominal muscle and the distances between the internal oblique muscle and the external oblique muscle were measured. The measurements were made at the same position in areas $(1 \mathrm{~cm}$ away from the center of the muscles) where the muscles are separated from their muscle fascias [15]. The measurement of the muscle thicknesses is presented in Figure 1.

The ratios of changes in the muscle thicknesses of the three abdominal muscles were determined by dividing the average thickness value measured in contracted states during each exercise by the average thickness measured in resting states.

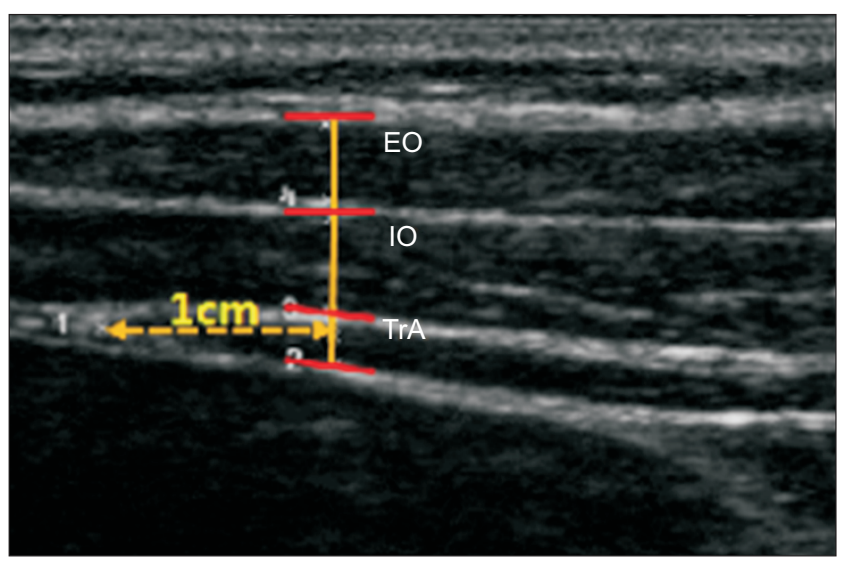

Figure 1. Ultrasound image of the abdominal muscles. E0, external oblique; 10, internal oblique; TrA, transverse abdominis. 


\section{Measurement Procedure}

In the present study, an ultrasonic diagnostic imaging system was used to measure the thickness of the abdominal muscle during the bridging exercises using the suspense sling system and balls with and without air cushions, which were used in addition to the slings and balls to provide different degrees of difficulties of tasks.

At that start of the bridging exercises, the subjects maximally bent their knee joints and placed both their arms on their normal anatomical position, with the palms of their hands facing down. Their knees and both feet were close together, and the soles of the feet were placed parallel on the ground. The subjects were instructed not to press the floor hard with their heads to ensure that their heads did not bear the weight. A blanket of the same height as the air cushion was placed between the scapular and C7. In the bridging exercises using a suspension sling system, the heights of the straps of the slings were determined so that the straps were at the knee level of individual subjects, and the straps were placed at points perpendicular to the suspension sling system. In the bridging exercises using balls, a $55 \mathrm{~cm}$ high ball was used.

The bridging exercises using slings and balls were divided into three stages (Figure 2). In the stage 1 bridging exercises, the sling straps and the ball were placed under the knee joints of each subject, and the subject was instructed to perform abdominal draw-in maneuvers. The subject was then instructed to perform a bridging exercise to place the knees, hips, and shoulders along a straight line and hold the posture for $5 \mathrm{sec}^{-}$ onds. In the stage 2 bridging exercises, in the starting position, the slings and the ball were placed below the ankle joint to lengthen the lever arm, and the same bridging exercise as performed in stage 1 was performed. In stage 3 , in the starting position, the straps of the sling and the ball were placed under the ankle joints as in stage 2 , and an air cushion was used instead of a blanket. Then, the subject performed the same bridging exercise as performed in stage 1 . All the subjects were educated about abdominal draw-in maneuvers and bridging exercises in each stage in advance. The order of the independent variables (two exercises and three stages) was determined via randomized control trials. A rest time of 1 minute was allowed between each experiment, and each experiment was repeated three times.

\section{Data Analysis}

In the present study, statistical analyses were conducted using the SPSS ver. 18.0 program (IBM Corp., Armonk, NY, USA). The ultrasound image show the means and standard deviations. Ratio of abdominal muscle thickness under independent variables (stages) were examined using One-way ANOVAs with repeated measures. The least significant difference was used as post hoc analyses. The level of statistical significance $\alpha$ was set to 0.05 .
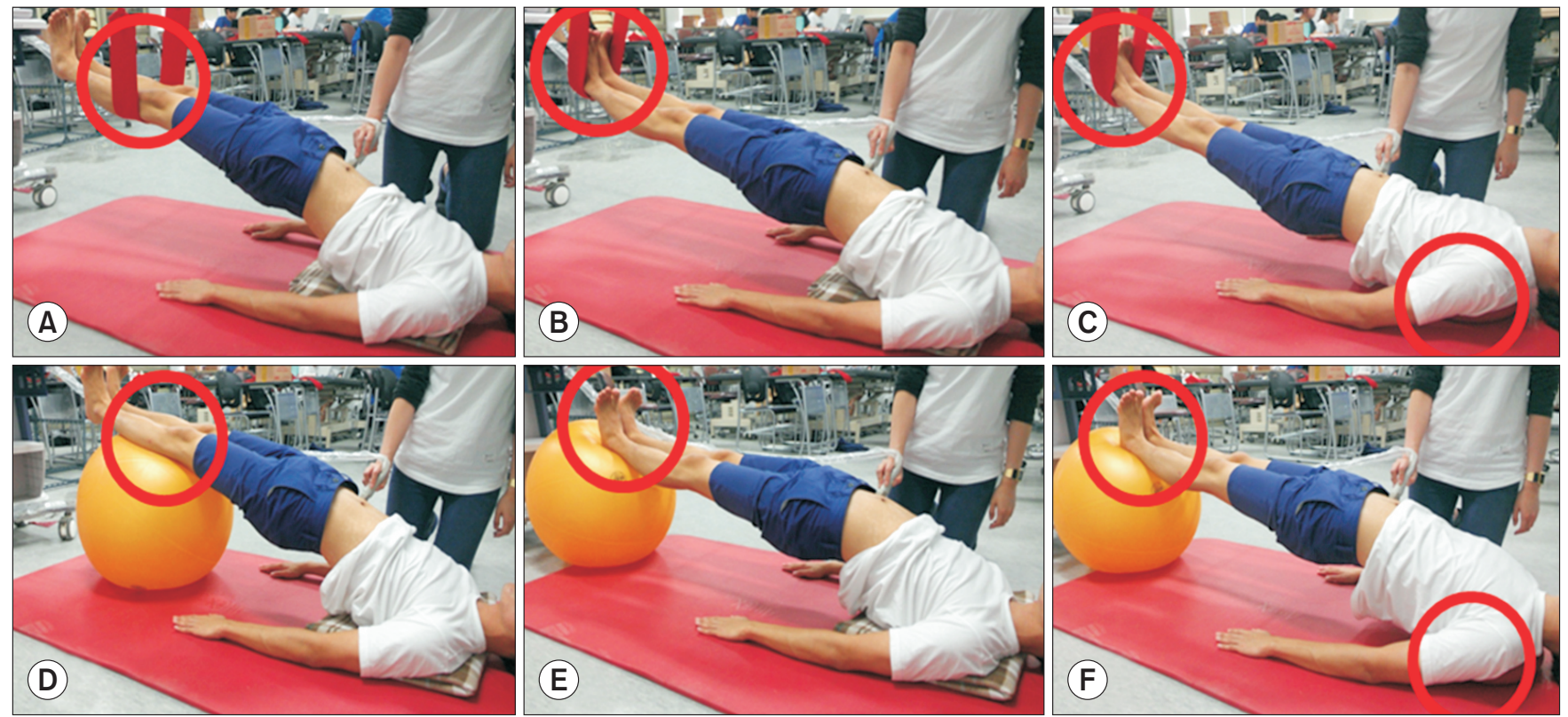

Figure 2. Bridging exercises using slings and balls. (A) Stage 1, (B) stage 2, (C) stage 3 of bridging exercises using slings and (D) stage 1, (E) stage 2, (F) stage 3 of bridging exercises using balls. 


\section{RESULTS}

\section{Thicknesses of the Abdominal Muscles at Different Levels of Difficulties during Bridging Exercises Using Slings And balls}

The thicknesses of the transverse abdominal muscle, the internal oblique muscle, and the external oblique muscle in each stage of the bridging exercises using slings and balls are shown in Table 1.

\section{Changes in the Thicknesses of Abdominal Muscles during Bridging Exercises Using Slings and Balls}

The muscle thickness ratios (mean contracted-rest thickness ratios) of the transverse abdominal muscle, the internal oblique muscle, and the external oblique muscle in each stage of the bridging exercises performed at different lever arm lengths and degrees of instability are shown in Table 2. Changes in the thicknesses of the abdominal muscles in each stage of the bridging exercises performed using slings and those performed using balls were compared. According to the results, there were no significant differences ( $p>0.05)$. In the muscle thickness ratios of the transverse abdominal muscle, the internal oblique muscle, and the external oblique muscle. Changes in the thicknesses of the abdominal muscles throughout individual stages of the bridging exercises performed using the slings and balls were compared. According to the results, the thicknesses of the transverse abdominal muscle and the internal oblique muscle were significantly higher in stage 2 compared to stage 1 and significantly higher in stage 3 compared to stages 1 and 2 ( $p<$ 0.05). However, the external oblique muscle did not show any significant differences between the stages $(p>0.05)$.

Table 1. Thickness of the abdominal muscle at different levels during bridging exercise $(\mathrm{N}=25)$

\begin{tabular}{lcccc}
\hline & Rest & Stage 1 & Stage 2 & Stage 3 \\
\hline Sling & & & & \\
TrA $(\mathrm{mm})$ & $2.52 \pm 0.39$ & $3.41 \pm 0.80$ & $4.02 \pm 0.76$ & $4.79 \pm 1.00$ \\
IO (mm) & $5.91 \pm 1.42$ & $7.51 \pm 2.32$ & $8.13 \pm 2.49$ & $8.88 \pm 2.38$ \\
EO (mm) & $4.02 \pm 1.23$ & $3.92 \pm 1.26$ & $3.81 \pm 1.25$ & $3.68 \pm 1.25$ \\
Ball & & & & \\
TrA (mm) & $2.52 \pm 0.39$ & $3.14 \pm 0.52$ & $3.90 \pm 0.64$ & $4.66 \pm 0.99$ \\
IO (mm) & $5.91 \pm 1.42$ & $7.43 \pm 2.31$ & $8.13 \pm 2.24$ & $9.12 \pm 2.96$ \\
EO (mm) & $4.02 \pm 1.23$ & $3.85 \pm 1.17$ & $3.69 \pm 1.12$ & $3.67 \pm 1.12$ \\
\hline
\end{tabular}

Values are presented as mean \pm standard deviation. TrA, transverse abdominis muscle; 10 , internal oblique muscle, EO,external oblique muscle.

\section{DISCUSSION}

In clinical settings, rather than rehabilitation training on stable surfaces, rehabilitation training on unstable surfaces is recommended for therapeutic exercises for re-education of damaged muscles. In particular, to improve lumbar stabilization, loads imposed on the abdominal muscles are increased by appropriately adjusting the level of difficulty during bridging exercises performed on unstable surfaces. However, studies of the degrees of contraction of the abdominal muscles during tasks of different degrees of difficulties due to the type of unstable surface are rare. Therefore, the present study aimed to examine change in the thicknesses of the abdominal muscles when the lever arm length and the degree of instability were changed. The results of the present study indicated that changes in the thicknesses of the deep abdominal muscles increased as the level arm length and the degree of instability increased, regardless of the type of unstable surfaces.

Bridging exercises are an efficient exercising method that can optimally contract the local muscles, such as the transverse abdominal muscle and the internal oblique muscle, in relation to the global muscles, such as the external oblique muscle. They are one type of lumbar stabilization exercise that can relieve lumbar instability and improve the trunk muscles' coactivation functions [16]. It has been reported that bridging exercises performed on unstable surfaces such as balls and slings stimulate proprioception receptors and increase muscle recruitment more so than the same exercises performed on stable surfaces. To increase the degree of difficulty, increasing the length of the lever arm or the degree of instability is

Table 2. Ratio of abdominal muscle thickness to resting condition at different exercise method ( $\mathrm{N}=25$ )

\begin{tabular}{lcccc}
\hline & ${\text { Stage } 1^{\mathrm{a}}}$ & Stage $2^{\mathrm{a}}$ & Stage $3^{\mathrm{a}}$ & $\mathrm{F}$ \\
\hline TrA & & & & \\
$\quad$ Sling & $1.35 \pm 0.21$ & $1.60 \pm 0.25^{\mathrm{b}}$ & $1.91 \pm 0.33^{\mathrm{b}, \mathrm{c}}$ & $52.684^{*}$ \\
$\quad$ Ball & $1.25 \pm 0.14$ & $1.56 \pm 0.23^{\mathrm{b}}$ & $1.86 \pm 0.38^{\mathrm{b}, \mathrm{c}}$ & $48.525^{*}$ \\
IO & & & & \\
$\quad$ Sling & $1.24 \pm 0.24$ & $1.38 \pm 0.31^{\mathrm{b}}$ & $1.52 \pm 0.32^{\mathrm{b}, \mathrm{c}}$ & $15.198^{*}$ \\
$\quad$ Ball & $1.27 \pm 0.28$ & $1.39 \pm 0.28^{\mathrm{b}}$ & $1.55 \pm 0.44^{\mathrm{b}, \mathrm{c}}$ & $9.411^{*}$ \\
$\quad$ EO & & & & \\
$\quad$ Sling & $0.99 \pm 0.18$ & $0.97 \pm 0.24$ & $0.93 \pm 0.22$ & 1.784 \\
$\quad$ Ball & $0.97 \pm 0.19$ & $0.94 \pm 0.20$ & $0.93 \pm 0.21$ & 0.722 \\
\hline
\end{tabular}

Values are presented as mean \pm standard deviation. TrA, transverse $a b-$ dominis muscle; 10 , internal oblique muscle, EO,external oblique muscle. ${ }^{a}$ There is no difference between sling and ball condition, ${ }^{b}$ significant difference in thickness ratio with stage $1,{ }^{c}$ significant difference in thickness ratio with stage $2 .{ }^{*} p<0.05$. 
recommended [11,17]. Therefore, the present study examined whether changes in the thicknesses of the abdominal muscles varied when the length of the lever arm and the degree of instability were changed during bridging exercises performed on balls or slings using an ultrasonic diagnostic imaging system that can accurately analyze the degree of contraction of the deep and superficial abdominal muscles.

According to the results of the present study, the thicknesses of the deep abdominal muscles significantly increased $(\mathrm{p}<$ 0.05 ) in both bridging exercises performed using the slings and balls, whereas the thicknesses of the superficial abdominal muscles did not show any significant differences $(\mathrm{p}>0.05)$. The present results are supported by a study that reported that bridging exercises performed on unstable surfaces, in this case air cushions and form rolls, were more effective than bridging exercises performed on stable surfaces but that the degrees of muscle activation under the two unstable conditions were similar [18].

To increase the difficulty levels of each of the bridging exercises, changes in the length of the lever arm and the degree of instability were controlled, and changes in the thicknesses of the abdominal muscles at different degrees of difficulties were compared. According to the results, changes in the thicknesses of the deep abdominal muscles significantly increased as the length of the lever arm and the degree of instability increased in all the bridging exercises using slings or balls. However, there were no significant changes in the thicknesses of the superficial abdominal muscles. Thus, unlike the superficial abdominal muscles, the deep abdominal muscles responded more sensitively to increases in the loads in the tasks and to the instability conditions. The neuromuscular system exerts greater proprioceptive demands under unstable conditions. With regard to the results of the present study, the deep postural muscles of the trunk are considered to have been activated because the instability increased when the unstable surfaces, together with the increased lengths of the lever arm, increased destabilizing torque, thereby making it difficult to maintain postures [19]. Previous studies reported that as the length of the lever arm is increased to enhance the difficulty level, the loads imposed on the abdominal region increase. Thus, increased lengths of the lever arm are effective in the activation of the deep abdominal muscles. Previous studies also reported that as instability conditions increase, the activation of the deep abdominal muscles increase, thereby supporting the findings of the present study $[10,20]$.

The present study has some limitations. First, as it involved only healthy young persons, it is difficult to generalize the results to those with lumbopelvic problems or lumbar region instability. Second, as it was limited to the transverse abdominal muscle, the internal oblique muscle, and the external oblique muscle abdominal muscle, the results cannot be applied to all trunk muscles. To address these limitations, future studies should include more trunk stabilization muscles, as well as patients with lumbopelvic problems.

\section{CONCLUSIONS}

According to the results of the present study, the thicknesses of the deep abdominal muscles, such as the transverse abdominal muscle and the internal oblique muscle, significantly increased $(p<0.05)$ in the both bridging exercises performed using slings and balls as the difficulty level increased, whereas the thicknesses of the external oblique muscles did not show any significant differences. When the bridging exercises performed under different unstable conditions using slings and balls were compared, the thicknesses of the transverse abdominal muscle, the internal oblique muscle, and the external oblique muscle did not show any significant differences ( $p$ > 0.05). The results suggest that the use of slings and the use of balls during bridging exercises are effective in activating the deep abdominal muscles. Adjusting the length of the lever arm and the degree of instability to vary the level of difficulties of bridging exercises in line with progress made in rehabilitation may help optimize exercises for patients with low back pain.

\section{CONFLICTS OF INTEREST}

No potential conflict of interest relevant to this article was reported.

\section{AUTHOR CONTRIBUTIONS}

Conceptualization: Jong-duk Choi. Data curation: Young Moon, Jong-duk Choi. Formal analysis: Young Moon, Jongduk Choi. Investigation: Young Moon. Methodology: Jongduk Choi. Project administration: Jong-duk Choi. Supervision: Jong-duk Choi. Validation: Jong-duk Choi. Visualization: Young Moon, Jong-duk Choi. Writing - original draft: Young 
Moon. Writing - review \& editing: Jong-duk Choi.

\section{ORCID}

Young Moon, https://orcid.org/0000-0001-6945-2433

\section{REFERENCES}

1. Panjabi MM. Clinical spinal instability and low back pain. J Electromyogr Kinesiol 2003;13(4):371-9.

2. Herrington L, Davies R. The influence of pilates training on the ability to contract the transversus abdominis muscle in asymptomatic individuals. J Bodyw Mov Ther 2005;9(1):527.

3. Standaert CJ, Weinstein SM, Rumpeltes J. Evidence-informed management of chronic low back pain with lumbar stabilization exercises. Spine J 2008;8(1):114-20.

4. Bergmark A. Stability of the lumbar spine. A study in mechanical engineering. Acta Orthop Scand Suppl 1989;230:1-54.

5. Behm DG, Anderson K, Curnew RS. Muscle force and activation under stable and unstable conditions. J Strength Cond Res 2002;16(3):416-22.

6. Hodges PW, Richardson CA. Inefficient muscular stabilization of the lumbar spine associated with low back pain. A motor control evaluation of transversus abdominis. Spine (Phila Pa 1976) 1996;21(22):2640-50.

7. Stevens VK, Coorevits PL, Bouche KG, Mahieu NN, Vanderstraeten GG, Danneels LA. The influence of specific training on trunk muscle recruitment patterns in healthy subjects during stabilization exercises. Man Ther 2007;12(3):271-9.

8. García-Vaquero MP, Moreside JM, Brontons-Gil E, PecoGonzález N, Vera-Garcia FJ. Trunk muscle activation during stabilization exercises with single and double leg support. J Electromyogr Kinesiol 2012;22(3):398-406.

9. Bjerkefors A, Ekblom MM, Josefsson K, Thorstensson A. Deep and superficial abdominal muscle activation during trunk stabilization exercises with and without instruction to hollow.
Man Ther 2010;15(5):502-7.

10. Vera-Garcia FJ, Grenier SG, McGill SM. Abdominal muscle response during curl-ups on both stable and labile surfaces. Phys Ther 2000;80(6):564-9.

11. Saliba SA, Croy T, Guthrie R, Grooms D, Weltman A, Grindstaff TL. Differences in transverse abdominis activation with stable and unstable bridging exercises in individuals with low back pain. N Am J Sports Phys Ther 2010;5(2):63-73.

12. McMeeken JM, Beith ID, Newham DJ, Milligan P, Critchley DJ. The relationship between EMG and change in thickness of transversus abdominis. Clin Biomech (Bristol, Avon) 2004;19(4):337-42.

13. Teyhen DS, Gill NW, Whittaker JL, Henry SM, Hides JA, Hodges P. Rehabilitative ultrasound imaging of the abdominal muscles. J Orthop Sports Phys Ther 2007;37(8):450-66.

14. Teyhen DS, Rieger JL, Westrick RB, Miller AC, Molloy JM, Childs JD. Changes in deep abdominal muscle thickness during common trunk-strengthening exercises using ultrasound imaging. J Orthop Sports Phys Ther 2008;38(10):596-605.

15. Mannion AF, Pulkovski N, Toma V, Sprott H. Abdominal muscle size and symmetry at rest and during abdominal hollowing exercises in healthy control subjects. J Anat 2008;213(2):173-82.

16. McGill S. Low back disorders: evidence-based prevention and rehabilitation. Champaign: Human Kinetics; 2002.

17. Kisner C, Colby LA. Therapeutic exercise: foundations and techniques. 5th ed. Philadelphia: F.A. Davis; 2007.

18. Hong Y, Kwon OY, Yi CH, Jeon H, Weon J, Park K. Effects of the support surface condition on muscle activity of abdominalis and erector spinae during bridging exercises. Phys Ther Korea 2010;17(4):16-25.

19. Anderson K, Behm DG. Trunk muscle activity increases with unstable squat movements. Can J Appl Physiol 2005;30(1):33-45.

20. Akuthota V, Nadler SF. Core strengthening. Arch Phys Med Rehabil 2004;85(3 Suppl 1):S86-92. 